\title{
Electromyography Activity of Vastus Lateralis Muscle During Whole-Body Vibrations of Different Frequencies
}

\author{
Marco Cardinale ${ }^{1}$ AND JON LiM² \\ ${ }^{1}$ Department of Biomedical Science, University of Aberdeen, Scotland; ${ }^{2}$ Northern State University, South Dakota.
}

\begin{abstract}
The aim of this study was to analyze electromyography (EMG) responses of vastus lateralis muscle to different whole-body vibration frequencies. For this purpose, 16 professional women volleyball players (age, $23.9 \pm 3.6$ years; height, $182.5 \pm 11.1 \mathrm{~cm}$; weight, $78.4 \pm 5.6 \mathrm{~kg}$ ) voluntarily participated in the study. Vibration treatment was administered while standing on a vibrating platform with knees bent at $100^{\circ}$ (Nemes Bosco-system, Rome, Italy). EMG root mean square (rms) and was recorded for 60 seconds while standing on the vibrating plate in the following conditions: no vibrations and 30-, 40-, and 50-Hz vibration frequencies in random order. The position was kept for 60 seconds in each treatment condition. EMGrms was collected from the vastus lateralis muscle of the dominant leg. Statistical analysis showed that, in all vibration conditions, average EMGrms activity of vastus lateralis was higher than in the no-vibration condition. The highest EMGrms was found at $30 \mathrm{~Hz}$, suggesting this frequency as the one eliciting the highest reflex response in vastus lateralis muscle during whole-body vibrations in half-squat position. An extension of these studies to a larger population appears worthwhile to further elucidate the responsiveness of the neuromuscular system to whole-body vibrations administered through vibrating platforms and to be able to develop individual treatment protocols.
\end{abstract}

Key Words: muscle tuning, neuromuscular responses

Reference Data: Cardinale, M., and J. Lim. Electromyography activity of vastus lateralis muscle during whole-body vibrations of different frequencies. J. Strength Cond. Res. 17(3):621-624. 2003.

\section{Introduction}

$\mathrm{M}$ echanical vibrations applied to the muscle belly or tendons have been shown able to elicit reflex muscle contractions (12). This neuromuscular response has been named "tonic vibration reflex" (TVR) and has been shown to be mediated by mono- and polysynaptic pathways $(8,19)$. Muscle spindle Ia reflexes have been indicated as the major determinant of this vibration-induced neuromuscular activation (5) leading to the TVR. Recent observations have shown the possibility of utilizing vibrations as a training tool in athletic settings. In fact, neuromuscular performance has been enhanced through the administration of vibration treatment (2). These improvements have been attributed to an enhancement of neural factors determining neuromuscular performance: recruitment, synchronization, inter- and intramuscular coordination and also proprioceptors' responses. In this connection, it should be noticed that vibrations have been shown to be effective in inducing improvements in vertical jumping ability (3) and in mechanical power of lower limbs in elite athletes (4). Moreover, studies conducted by Issurin et al. $(13,14)$ have shown increases in explosive strength and flexibility in athletes. Vibrations applied to the arm showed enhancement of mechanical power and an increase in neuromuscular efficiency as indicated by a decrease in EMG/power ratio supporting the evidence that vibrations represent a strong stimulus for the neuromuscular system (2). Furthermore, EMG activity during vibrations has been shown to reach values higher than $200 \%$ of the baseline in arm flexor muscles (2). Previous studies have found vibrations determining an increased EMG activity in the muscles undergoing the vibration treatment $(15,7)$.

Vibrations are starting to be used as an alternative training means for enhancing strength/power characteristics. However, it should be pointed out that there is a lack of information on the effectiveness of different vibration frequencies on neuromuscular performance. Moreover, it should also be considered that currently there is no methodology able to identify the individual vibration load that an individual can sustain. Muscle activation during vibration can be monitored recording the EMG signal of the target muscles. With this tool, it is in fact possible to determine muscle activity in a given task. In light of the above, it is possible to affirm that EMG can be used to provide an indication of the muscle activity determined by vibration. In fact, the EMG signal can be used to measure 
the severity of muscle activation following the application of vibration. The aim of this study was to analyze EMG responses in the vastus lateralis muscle while standing on a vibrating plate producing oscillations of different frequencies to verify the hypothesis that different vibration frequencies determine different neuromuscular responses.

\section{Methods}

\section{Subjects}

Sixteen professional women volleyball players volunteered as subjects for the present study (age, $23.5 \pm$ 4.6 years; height, $183.4 \pm 8.4 \mathrm{~cm}$; weight, $75.1 \pm 7.4$ $\mathrm{kg}$ ). All of the subjects had competed for several years at a high level and were regularly training at the time of the experiment. Full advice was given to the volunteers regarding the possible risk and discomfort that might be involved, and all the subjects gave their written informed consent, approved by the ethical committee of the Italian Society of Sport Science, to participate in the experiment. Subjects with a previous history of fractures or bone injuries were excluded from the study.

\section{EMG Analysis}

The signals from the vastus lateralis of the dominant leg were recorded with bipolar surface electrodes (interelectrode distance, $1.2 \mathrm{~cm}$ ) including an amplifier (gain, 600; input impedance, $2 \mathrm{G} \Omega$; CMMR, $100 \mathrm{~dB}$; band-pass filter, 6-1500 Hz; Biochip, Grenoble, France) fixed longitudinally over the muscle belly. The MuscleLab converted the amplified EMG raw signal to an average root-mean-square (rms) signal via its built-in hardware circuit network (frequency response, $450 \mathrm{kHz}$; averaging constant, 100 milliseconds; total error, $\pm 0.5 \%$ ). The EMGrms was expressed as a function of the time (millivolts or microvolts). EMG cables were secured with an appropriate setup to prevent the cables from swinging and from causing movement artifact. A personal computer (PC Celeron 400) and the MuscleLab software were used to collect and store the data. The EMGrms was collected during each repetition, lasting 1 minute each, of isometric half squat. In a previous study, the reliability of the EMG measurements was shown to be 0.91 (2).

\section{Treatment Procedures}

Subjects were exposed to a vibration treatment (VT) using a vibration platform called Nemes Bosco-system (OMP, Rieti, Italy). The amplitude allowed by the vibration platform was (peak-to-peak) $10 \mathrm{~mm}$. The subjects were exposed randomly to three different VTs. The frequencies used in the experiment were 30, 40, and $50 \mathrm{~Hz}$. Each vibration treatment lasted 60 seconds, with 60 seconds of rest allowed between each VT frequency. The subjects were asked to stand in half-squat position on the vibration platform (knee angle $100^{\circ}$ ) as

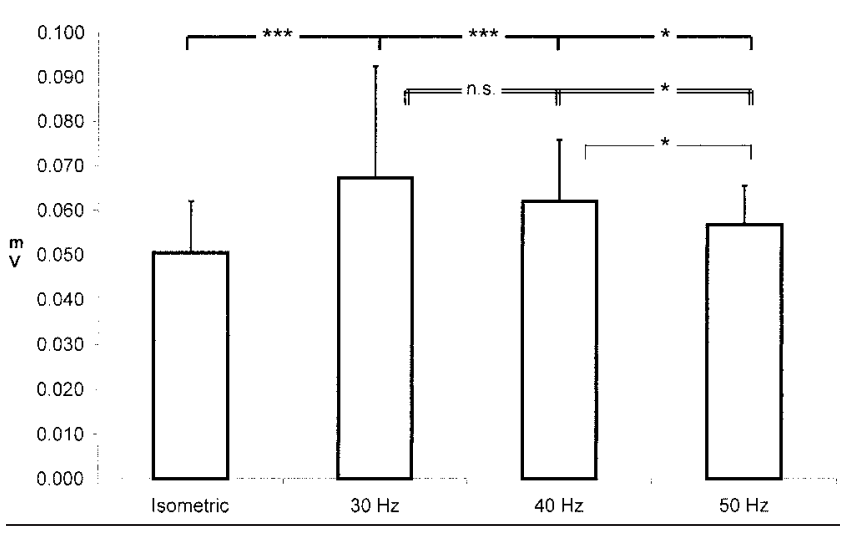

Figure 1. Electromyography root mean square (EMGrms) average values of vastus lateralis recorded during different vibration frequencies. The error bars indicate standard deviation. ${ }^{* *}=p<0.001 ;{ }^{*}=p<0.05 ;$ n.s. $=$ nonsignificant.

indicated in previous studies (3). The EMG signal was collected from the vastus lateralis muscle during the 60 -second duration of the testing position. A total of 4 sets lasting 60 seconds each were performed with 60 seconds of rest between sets allowed. EMGs were recorded for 5 seconds before starting the vibration treatment to verify the absence of residual muscle activity. If the EMG was different from the baseline measurement, a further 30 seconds of rest were allowed. The subjects then performed the isometric half squat in the following conditions: no vibrations, and randomly $30-, 40-$, and 50-Hz vibration frequency.

\section{Statistical Analyses}

Ordinary statistical methods were employed, including means $(X)$ and standard deviation $( \pm S D)$. Average EMGrms values for vastus lateralis were considered for analysis. To analyze differences in EMG between vibration frequencies, a repeated measures ANOVA was computed to identify significant differences for the dependent variables. Significant $F$ values were followed by paired $t$-tests for within- and between-group comparisons. Significance was set at $p \leq 0.05$.

\section{Results}

Whole-body vibration treatment lead to an increase of EMGrms activity of vastus lateralis muscle as compared with baseline values $(p<0.001)$ collected in the no-vibration condition (see Figure 1). The highest EMGrms activity was found at $30-\mathrm{Hz}$ vibration frequency $(+34 \%, p<0.001)$. Between-treatment comparisons showed statistically significant differences between $30-$ and $50-\mathrm{Hz}$ frequencies $(20 \%, p<0.05)$ and $40-$ and $50-\mathrm{Hz}$ frequencies $(10 \%, p<0.05)$. EMGrms activity between $30-$ and $40-\mathrm{Hz}$ vibration frequencies did not show any statistically significant difference $(9 \%$, n.s.). 


\section{Discussion}

The results of the present study demonstrated an increased EMG activity in vastus lateralis muscle at various vibration frequencies when subjects were standing on a vibration platform. An increase in EMG has been observed in quadriceps muscle undergoing vibrations and it has been attributed to a facilitation of the excitability of spinal reflex (7). Based on these findings, it was possible to verify that whole-body vibrations transmitted through a vibrating platform in halfsquat position were able to determine a higher EMG activity compared with the nonvibrating condition. This effect has been related to excitation of primary endings of muscle spindles and activation of $\alpha$-motoneurons as indicated elsewhere (i.e., 12, 16).

In our opinion, two factors could account for the observed increase in EMG activity: i) the initial length of analyzed muscles and ii) the frequency of vibratory stimulation. In fact, it is already known that vibratory stimulation is more effective in stretched muscles (21, 9). Vibration sensitivity of human muscle spindles has also been demonstrated in single human spindle afferent by Burke and Gandevia (5). These observations support the utilization of the half-squat position on the vibrating platform as an effective position for triggering vastus lateralis stimulation. Escamilla et al. (10) reported that the two vasti muscles produce $40-50 \%$ more activity than the rectus femuris during half squat. Moreover, compared with each other, the vastus medialis and vastus lateralis produce approximately the same amount of activity $(10,11,24)$. The position chosen in the experiment could then be considered optimal for stimulating the vastus lateralis muscle because of the lengthened position and the activation relative to the quadricep muscles, as suggested elsewhere (1).

Vibrations-induced increases in EMG activity and the consequent degree of motor unit synchronization have been shown to be dependent on the vibration frequency $(6,17,19,23)$. This was not observed in our experiment because the highest EMGrms activity was found when the frequency was $30 \mathrm{~Hz}$. However, previous observations at constant displacement amplitude have shown that monosynaptic inhibition does not vary with vibration frequency (18). The missing EMG augmentation with vibration frequency may be due to inhibitory mechanisms mediated by mechanoceptors and skin receptors, which have been shown to be activated during whole-body vibrations and contribute to the EMG activity (22). The results of our study suggest that, in the specific position used, the frequency able to cause the highest EMG response in vastus lateralis muscle was $30 \mathrm{~Hz}$. In our opinion, vibrations are strong perturbations that are perceived by the central nervous system, which modulates the stiffness of the stimulated muscle groups. The reflex muscle activ- ity could then be considered a neuromuscular tuning response to minimize soft-tissue vibrations. These responses are individual and probably could be population-specific and could be based on mechanical and reflex factors. Natural frequencies of muscle groups in athletes' legs have been reported to be between 5 and $65 \mathrm{~Hz}$ (20); the input frequencies used in our study are in this range and it suggests that individual responses could be related to individual capabilities in damping external perturbations to avoid resonance effects. These adaptations have been hypothesized during running in humans (20), and our opinion is that the same principles apply to vibrations superimposed with vibrating plates.

In conclusion, this study indicates, first, that standing on a vibrating platform in half-squat position elicits higher EMG responses in vastus lateralis muscle as compared with the same position without vibrations being transmitted. Second, EMG recordings could represent a means for individualizing training protocols for whole-body vibrations. Further studies are needed for elucidating the mechanisms determining individual responses and the effectiveness of individualized treatments on neuromuscular performance.

\section{Practical Applications}

Vibrations exercise has been shown to be effective in enhancing strength/power capabilities (i.e., 2-4, 13, 14). However, it should be pointed out that there is no current knowledge about effective exercise protocols or measurements to which to refer when prescribing a vibration exercise program. One way for individualizing vibration treatments could be the use of surface EMG to assess muscle responsiveness to different vibration frequencies. In fact, the results of this study support the idea that vibrations elicit higher EMG activity compared with isometric conditions. Also, different vibration frequencies elicit different EMG responses in the stimulated muscles.

This new technique could be used separately or in combination with conventional strength-training routines. In particular, due to the effects of vibration on stretch reflexes, it can be suggested for use in a complex training-type routine in place of plyometric drills. Vibration in fact stimulates reflex muscle responses due to the small and quick changes in length of the muscle-tendon complex and the small and fast joint rotations associated with the oscillatory motion. Those stimuli are similar to the ones experienced during plyometric exercise and place less stress on joints due to the reduced impact load. Future studies are needed in order to develop safe exercise procedures on vibrating platforms and in order to understand the effectiveness of different vibration exercise protocols. 


\section{References}

1. Anderson, R., C. Courtney, And E. Carmeli. EMG analysis of the vastus medialis/lateralis muscles utilizing the unloaded narrow-and wide-stance squats. J. Sports Rehab. 7:236-247. 1998.

2. Bosco, C., M. Cardinale, and O. Tsarpela. Influence of vibration on mechanical power and electromyogram activity in human arm flexor muscles. Eur. J. Appl. Physiol. 79:306-311. 1999.

3. Bosco, C., M. Cardinale, O. Tsarpela, R. Colli, J. Tihany, S.P. VON DUVILlard, AND A. VIRU. The influence of whole body vibration on jumping performance. Biol. Sport 15:157-164. 1998.

4. Bosco, C., R. Colli, E. Introini, M. Cardinale, O. Tsarpela, A. Madella, J. TihanyI, AND A. Viru. Adaptive responses of human skeletal muscle to vibration exposure. Clin. Physiol. 19: 183-187. 1999.

5. BurKe, D., AND S.C. GANDEVIA. The human muscle spindle and its fusimotor control. In: Normal Control of Movement. W.Q. Ferrel and U. Proske, eds. New York: Plenum Press, 1995. pp. $19-25$.

6. BURKE, J.R., W.Z. RYMER, AND H.V. WALSH. Relative strength of synaptic inputs from short latency pathways to motor units of defined type in cat medial gastrocnemious. Neurophysiology 39:447-458. 1976.

7. BurKe, J.R., M.C. Schutten, D.M. KocejA, AND G. KAMEN. Age-dependent effects of muscle vibration and the Jendrassik manoeuvre on the patellar tendon reflex response. Arch. Phys. Med. Rehabil. 77(6):600-604. 1996.

8. Desmedt, J.E., AND E. Godeaux. The tonic vibration reflex and the vibration paradox in limb and jaw muscle in man. In: Spinal and Supraspinal Mechanisms of Voluntary Motor Control and Locomotion. J.E. Desmedt, ed. Basel: Karger, 1980. pp. 215-242.

9. EKLUND, G., AND K.E. Hagbarth. Normal variability of tonic vibration reflexes in man. Exp. Neurol. 16:80-92. 1966.

10. Escamilla, R.F., G.S. Fleisig, N. Zheng, S.W. Barrentine, K.E. WILK, AND J.R. ANDREWS. Biomechanics of the knee during closed kinetic chain and open kinetic chain exercises. Med. Sci. Sports Exerc. 30:556-569. 1998.

11. Escamilla, R.F., N. Zheng, G.S. Fleisig, J.E. Lander, S.W. BarRENTINE, G.R. CUTTER, AND J.R. ANDREWS. The effects of technique variations on knee biomechanics during squat and leg press. Med. Sci. Sports Exerc. 29:S156. 1997.

12. Hagbarth, K.E., AND G. EKLUnd. Motor effects of vibratori stimuli in man. In: Proceedings of the First Nobel Symposium on Muscular Afferents and Motor Control. R. Granit, ed. Stockholm: Almqvist and Wiksell, 1965. pp. 177-186.

13. Issurin, V.B., D.G. LiebermanN, AND G. Tenenbaum. Effect of vibratory stimulation training on maximal force and flexibility. J. Sport Sci. 12:562-566. 1994.

14. Issurin, V.B., AND G. Tenenbaum. Acute and residual effects of vibratory stimulation on explosive strength in elite amateur athletes. J. Sport Sci. 17:177-182. 1999.

15. LANCE, J.W., D.J. BURKE, AND C.J. ANDREWS. The reflex effects of muscle vibrations. In: New Developments in Electromyography and Clinical Neurophysiology (vol. 3). J.E. Desmedt, ed. Basel: Karger, 1973. pp. 444-462.

16. Lebedev, M.A., AND A.V. Peliakov. Analysis of the interference electromyogram of human soleus muscle after exposure to vibration. Neirofiziologia 23:57-65 (article in Russian, summary in English). 1991.

17. MARTIN, B.J., AND H.S. PARK. Analysis of the tonic vibration reflex: Influence of vibration variables on motor unit synchronization and fatigue. Eur. J. Appl. Physiol. 75:504-511. 1997.

18. Martin, B.J., J.P. RolL, AND G.M. GAUTHIER. Inhibitory effects of combined agonist and antagonist vibration on H-reflex. Aviat. Space Environ. Med. 57:681-687. 1986.

19. Matthews, P.B.C. The reflex excitation of soleus muscle of the decerebrate cat caused by vibration applied to its tendon. J. Physiol. 184:470-472. 1966.

20. NIGG, B.M., AND J.M. WAKELING. Impact forces and muscle tuning: A new paradigm. Exerc. Sport Sci. Rev. 29:37-41. 2000.

21. NoRdin, M., AND K.E. HAGBARTH. Effects of preceding movements and contractions on the tonic vibration reflex of human finger extensor muscles. Acta Physiol. Scand. 156:435-440. 1996.

22. Ribot-Ciscar, E., J.P. Vedel, AND J.P. Roll. Vibration sensitivity of slowly and rapidly adapting cutaneous mechanoceptors in the human foot and leg. Neurosci. Lett. 104:130-135. 1989.

23. RolL, J.P., J.P. VedEL, AND E. RiBOT. Alteration of proprioceptive messages induced by tendon vibration in man: A microneurographic study. Exp. Brain. Res. 76:213-222. 1989.

24. Signorile, G.F., B. Weber, B. Roll, J.F. Caruso, I. LowenSTEYN, AND A.C. PERRY. An electromyographical comparison of the squat and knee extension exercises. J. Strength Cond. Res. 8:178-183. 1994.

Address correspondence to Marco Cardinale, m.cardinale@abdn.ac.uk. 